\title{
ON THE MONODROMY OF HIGHER LOGARITHMS
}

\author{
DINAKAR RAMAKRISHNAN
}

\begin{abstract}
The (multivalued) higher logarithms are interpreted, by studying their monodromy, as giving well-defined maps from $\mathbf{P}_{\mathbf{C}}^{1}-\{3$ points $\}$ into certain complex nilmanifolds with $\mathbf{C}^{*}$-actions.
\end{abstract}

The purpose of this note is to exhibit a family of unipotent representations of $\mathbf{Z} * \mathbf{Z}$ arising naturally from the monodromy of the higher logarithms $\ln _{k}$ (see [4]), and thereby interpret each $\ln _{k}$ as yielding a well-defined map $\rho_{k}$ of $\mathbf{P}_{\mathbf{C}}^{1}-\{0,1, \infty\}$ into a $(k+1)$-dimensional complex nilmanifold $\boldsymbol{M}_{k+1}$ equipped with a $\mathbf{C}^{*}$-action. Moreover, a natural holomorphic connection $\nabla_{k}$ is shown to exist on each fibration $M_{k+1} \rightarrow M_{k+1} / C^{*}$, with respect to which $\rho_{k}$ is flat. The role of dilogarithm in the study of volumes of hyperbolic 3-manifolds [5], arithmetic [1,2], $K$-theory and Kac-Moody Lie algebras [3] leads one to hope for such interesting links in the case of higher logarithms as well. Furthermore, the tower of nilmanifolds associated to $\mathbf{P}_{\mathbf{C}}^{1}-\{3$ points $\}$ via $\left\{\ln _{k}\right\}$ suggests, following a remark of $\mathbf{P}$. Deligne, relations to Sullivan's theory of differential forms. We hope to pursue this at some future time. Finally, we have come to learn recently of an independent and very elegant construction of these nilmanifolds due to J. W. Milnor (unpublished).

We would like to thank Spencer Bloch for his constant and friendly encouragement.

For $x$ in $C$, set $\ln _{0}(x)=x /(1-x)$ and $L(x)=(2 \pi i)^{-1} \log (x)$. Then $\ln _{k}$ is defined recursively by

$$
\ln _{k}(x)=\int_{0}^{x} \ln _{k-1}(t) d L(t)
$$

It follows easily that each $\ln _{k}$ satisfies the $(k+1)$ st order, homogeneous, algebraic differential equation

$$
\frac{d}{d x}\left((1-x) \frac{d}{d x}\left(\left(x \frac{d}{d x}\right)^{k-1} U\right)\right)=0
$$

This equation has regular singular points at 1 and $\infty$ if $k=1$, and at 0,1 , and $\infty$ if $k>1$.

Received by the editors August 3, 1981.

1980 Mathematics Subject Classification. Primary 53C30, 22E40; Secondary 33A75.

(C)1982 American Mathematical Society 0002-9939/82/0000-1081/\$02.00 
Now let, for each integer $m>2$,

$$
N_{m}=\left\{\left(\begin{array}{ccc}
1 & & * \\
& \ddots & \\
0 & & 1
\end{array}\right)\right\} \subset \mathrm{GL}_{m}, \quad U_{m}=\left\{\left(\begin{array}{ccc}
1 & 1 & * \\
0 & 1 & - \\
0 & I
\end{array}\right)\right\},
$$

and let [ $]_{m}$ denote the one-parameter subgroup $\mathbf{G}_{a} \rightarrow N_{m}$ given by

$$
y \mapsto\left(\begin{array}{ccccccc}
1 & 0 & & & & \cdots & 0 \\
& 1 & y & y^{2} / 2 ! & y^{3} / 3 ! & \cdots & y^{(m-1)} /(m-1) ! \\
& & 1 & y & y^{2} / 2 ! & \cdots & y^{(m-2)} /(m-2) ! \\
& & & 1 & \cdots & \vdots \\
& & & & \cdots & y \\
0 & & & & & & 1
\end{array}\right)
$$

The image of [ $]_{m}$ normalizes $U_{m}$. Let $R_{m}$ denote the corresponding semidirect product. It is then an algebraic subgroup of $N_{m}$ isomorphic to $\mathbf{G}_{a} \ltimes \mathbf{G}_{a}^{(m-1)}$. We will write the elements of $R_{m}$ as $\left([y]_{m} ;\left(x_{1}, \ldots, x_{m-1}\right)\right)$ with $y, x_{i}$ in $\mathbf{G}_{a}$. Let $R_{2}$ denote $\mathbf{G}_{a} \times \mathbf{G}_{a}$.

Let $\alpha$ and $\beta$ denote respectively the cycles around 0 and 1 in $\mathbf{P}_{\mathbf{C}}^{1}-\{0,1, \infty\}$, oriented in the usual way. Then $\pi_{1}=\pi_{1}\left(\mathbf{P}_{\mathbf{C}}^{1}-\{0,1, \infty\}\right)$ is a free group on $\alpha$ and $\beta$. Now for each $k \geqslant 1$, define a representation $\lambda_{k}: \pi_{1} \rightarrow R_{k+1}(C) \subset G L_{k+1}(C)$ by

$$
\alpha \mapsto\left([1]_{k+1} ;(0, \ldots, 0)\right) \text { and } \beta \mapsto\left([0]_{k+1} ;(1,0, \ldots, 0)\right) \text {. }
$$

Put $\Gamma_{k+1}=\lambda_{k}\left(\pi_{1}\right)$. It is a discrete, $k$-step nilpotent subgroup of $R_{k+1}(C)$. Note that $\Gamma_{3}$ is the $Z$-points of the 3-dimensional Heisenberg group and that $\Gamma_{2}=Z^{2}=$ $\pi_{1}^{a b}=\Gamma_{k+1}^{a b}$, for every $k \geqslant 1$.

Let $M_{k+1}$ denote the complex nilmanifold $\Gamma_{k+1} \backslash R_{k+1}(\mathrm{C})$.

THEOREM. (a) The (multivalued map) $\rho_{k}: \mathbf{P}_{\mathbf{C}}^{1}-\{0,1, \infty\} \rightarrow R_{k+1}$ (C) $\subset N_{k+1}$, given by

$$
x \mapsto\left([L(x)]_{k+1} ; \ln _{1}(x), \ln _{2}(x), \ldots, \ln _{k}(x)\right),
$$

becomes well defined modulo $\Gamma_{k+1}$.

(b) Each $M_{k+1}$ comes equipped with a $\mathbf{C}^{*}$-action with the quotient being identified with $M_{k}$. If $p_{k}$ denotes the corresponding projection $M_{k+1} \rightarrow M_{k}$, then we have the commutative diagram ( for $k \geqslant 2$ )

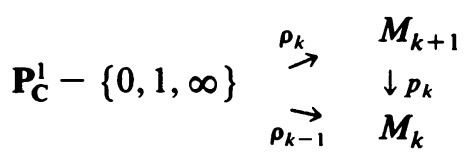

(c) There exists a holomorphic connection $\nabla_{k}$ on $M_{k+1} \stackrel{p_{k}}{\rightarrow} M_{k}$ such that $\rho_{k}$ yields a flat section to the corresponding pullback (via $\rho_{k-1}$ ) bundle with connection on $\mathbf{P}_{\mathbf{C}}^{1}-\{0,1, \infty\}$.

Proof. (a) The solution space to $(*)_{k}$ is spanned by $\left\{\ln _{k}, L^{j} / j ! \mid 0 \leqslant j \leqslant k-1\right\}$. It is easy to check that the monodromy around 1 amounts to sending $\ln _{k}$ to 
$\ln _{k}+L^{(k-1)} /(k-1)$ ! and fixing the $\left(L^{j} / j !\right)$ 's. Consequently, $\beta$ acts on

$$
v_{k} \stackrel{\text { def }}{=}\left(\begin{array}{c}
\ln _{k} \\
L^{(k-1)} /(k-1) ! \\
\vdots \\
L \\
1
\end{array} \mid\right.
$$

by multiplication on the left by $\rho_{k}(\beta)$.

The monodromy around 0 fixes $\ln _{k}$ and sends each $L^{j} / j$ ! to

$$
\sum_{0<i<j} \frac{1}{(j-i) !} \frac{L^{i}}{i !} \text {. }
$$

Thus $\alpha$ acts on $v_{k}$ via $\rho_{k}(\alpha)$ on the left. The assertion (a) now follows, since $\lambda_{k}$ sends $x$ to the matrix whose $(l+1)$ st column is

$$
\left.\left(\begin{array}{c}
v_{l}(x) \\
1 \\
0
\end{array}\right)\right\} k-l .
$$

(b) Define $p_{k}: R_{k+1} \rightarrow R_{k}$ when $k>2$ (resp. $\left.k=2\right)$ by

$$
\left([y]_{k+1} ;\left(x_{1}, \ldots, x_{k}\right)\right) \rightarrow\left([y]_{k} ;\left(x_{1}, \ldots, x_{k-1}\right)\right)
$$

(resp. $\left.\left([y]_{3} ;\left(x_{1}, x_{2}\right)\right) \rightarrow\left(y, x_{1}\right)\right)$.

In either case let $i_{k}$ denote the injection $\mathbf{G}_{a} \rightarrow R_{k+1}$ given by $z \rightarrow$ $\left([0]_{k+1} ;(0, \ldots, 0, z)\right)$. Then we have the exact sequence

$$
0 \rightarrow \mathbf{G}_{a} \stackrel{i_{k}}{\rightarrow} R_{k+1} \stackrel{p_{k}}{\rightarrow} R_{k} \rightarrow 1 .
$$

Note that since $\Gamma_{k+1}$ is a discrete, $k$-step nilpotent subgroup of $R_{k+1}(C)$, the intersection of $i_{k}(\mathbf{Q})$ with $\Gamma_{k+1}$ is a rank-1 free abelian group, and is generated by $i_{k}\left(t_{k}\right)$ for some $t_{k}$ in $\mathbf{Q}$.

The map $p_{k}$ makes sense on $\Gamma_{k+1}$ and $p_{k}\left(\Gamma_{k+1}\right)=\Gamma_{k}$. Consequently we have the commutative exact diagram

$$
\begin{aligned}
& 0 \rightarrow \mathrm{C} \stackrel{\stackrel{i_{k}}{\rightarrow}}{\rightarrow} \boldsymbol{R}_{k+1}(\mathrm{C}) \stackrel{p_{k}}{\rightarrow} \boldsymbol{R}_{k}(\mathrm{C}) \rightarrow 1 \\
& 0 \rightarrow t_{k} \mathbf{Z} \stackrel{i_{k}}{\rightarrow} \Gamma_{k+1} \stackrel{p_{k}}{\rightarrow} \quad \Gamma_{k} \rightarrow 1 .
\end{aligned}
$$

Identifying $\mathbf{C} / t_{k} \mathbf{Z}$ with $\mathbf{C}^{*}$ via $z \mapsto \exp \left(2 \pi i z / t_{k}\right)$, we get an action of $\mathbf{C}^{*}$ on each $M_{k+1}=\Gamma_{k+1} \backslash R_{k+1}(\mathrm{C})$. The quotient clearly identifies, via $p_{k}$, to $\boldsymbol{M}_{k}=\Gamma_{k} \backslash R_{k}(\mathrm{C})$. Finally, unwinding the definition of $\left\{\rho_{k}\right\}$ we see that $\rho_{k-1}=p_{k} \circ \rho_{k}$.

(c) Let $G_{k+1}$ (resp: $C_{k+1}$ ) denote the complex Lie group $i_{k}\left(t_{k} Z\right) \backslash R_{k+1}(\mathrm{C})$ (resp. $i_{k}\left(t_{k} Z\right) \backslash i_{k}(\mathbf{C})$ ), and let $\tilde{\Gamma}_{k+1}$ denote the image of $\Gamma_{k+1}$ under the canonical projection $R_{k+1}(C) \rightarrow G_{k+1}$ : Then $M_{k+1}$ is none other than the quotient of $G_{k+1}$ by the left action of $\tilde{\Gamma}_{k+1}$. If $\Delta_{k+1}$ denotes the group $\tilde{\Gamma}_{k+1} \cdot C_{k+1}$ in $\tilde{G}_{k+1}$, then there exists 
a unique character $\mu_{k+1}$ of $\Delta_{k+1}$ which is trivial on $\tilde{\Gamma}_{k+1}$ and is the identity on $C_{k+1}$. The space $\mathcal{L}_{k}$ of holomorphic sections of the line bundle associated to $p_{k}: M_{k+1} \rightarrow M_{k}$ can now be identified (as a right $G_{k+1}$-module) with the representation of $G_{k+1}$ induced holomorphically by $\mu_{k+1}$. This gives rise to an action $\pi_{k}$ of $\operatorname{Lie} G_{k+1}$ on $\mathcal{L}_{k}$. To define a connection we have to give a way to differentiate the sections in $\mathcal{L}_{k}$ by the derivations on the base $M_{k}$. To do this we first note that Lie $G_{k+1}$ can be realized, when $k>2$ (resp. $k=2$ ), as

$$
\left\{\left[y ; x_{1}, x_{2}, \ldots, x_{k}\right]=\left|\begin{array}{ccccc}
0 & x_{1} & x_{2} & \cdots & x_{k} \\
& 0 & y & \cdots & y \\
& & 0 & \ddots & \vdots \\
& & & \ddots & y \\
0 & & & & 0
\end{array}\right| \mid x_{1}, \ldots, x_{k}, y \in \mathbf{C}\right\}
$$

(resp. $\{(y, x) \mid y, x \in \mathbf{C}\}$ ). We see that the map Lie $G_{k+1} \rightarrow$ Lie $G_{k}$ (coming from the differential of $p_{k}$ ) admits a vector space- (but not a Lie algebra-) section $s_{k}$ given, when $k>2$ (resp. $k=2$ ), by $\left[y ; x_{1}, \ldots, x_{k-1}\right] \mapsto\left[y ; x_{1}, \ldots, x_{k-1}, 0\right]$ (resp. $(y, x) \mapsto[y ; x, 0])$. Now define $\nabla_{k}$ to be the holomorphic connection defined by the action on $\mathcal{L}_{k}$ via $\pi_{k} \circ s_{k}$ of the right invariant derivations on $G_{k}$.

It is a simple exercise to verify that, locally on $\mathbf{P}_{\mathbf{C}}^{1}-\{0,1, \infty\}$, a section $\varphi$ to the pullback bundle with connection via $\rho_{k-1}$ is flat if and only if

$$
\varphi \cdot \ln _{k-1}(x) d L(x)+d \varphi=0 .
$$

Certainly, $\rho_{k}$ gives rise to such a (flat) section. Q.E.D.

\section{REFERENCES}

1. A. A. Beilinson, Higher regulators and values of L-functions of curves, Functional Anal. Appl. 14 (1980), 116-118.

2. S. Bloch, Lectures on algebraic cycles, Duke Univ. Math. Ser., vol. IV, Department of Mathematics, Duke University, Durham, N.C., 1980.

3. __ Dilogarithm and extensions of Lie algebras, Algebraic K-Theory, (M. Stein and E. Friedlander, eds.),Springer-Verlag, New York, 1981.

4. L. Lewin, Polylogarithms and associated functions, North-Holland, Amsterdam, 1981.

5. W. Thurston, Geometry and topology of 3-manifolds, Chapter 7 by J. W. Milnor, Lecture notes, Princeton University.

Department of Mathematics, University of Chicago, Chicago, Illinois 60637 